\title{
HSP90 as a novel molecular target in non-small- cell lung cancer
}

\author{
This article was published in the following Dove Press journal: \\ Lung Cancer: Targets and Therapy \\ I March 2016 \\ Number of times this article has been viewed
}

\section{Khashayar Esfahani \\ Victor Cohen}

Segal Cancer Center, Jewish General Hospital, McGill University, Montreal, QC, Canada

Correspondence: Victor Cohen Segal Cancer Center, Jewish General Hospital, McGill University, 3755 Chemin de la Côte-Sainte-Catherine, Montreal, QC, H3T IE2, Canada Email vcohen@jgh.mcgill.ca
Abstract: Lung cancer remains the most lethal cancer, with over 160,000 annual deaths in the USA alone. Over the past decade, the discovery of driver mutations has changed the landscape for the treatment of non-small-cell lung cancer (NSCLC). Targeted therapies against epidermal growth factor receptor (EGFR) or anaplastic lymphoma kinase (ALK) have now been approved by the Food and Drug Administration as part of the standard first-line treatment of NSCLC. Despite good initial responses, most patients develop resistance within 8-12 months and have disease progression.

Keywords: non-small-cell lung cancer, driver mutations, targeted therapy, heat shock protein 90 (HSP90)

\section{Introduction}

Lung cancer remains the most lethal cancer, with over 160,000 annual deaths in the USA alone. ${ }^{1}$ Over the past decade, the discovery of driver mutations has changed the landscape for the treatment of non-small-cell lung cancer (NSCLC). ${ }^{2}$ Targeted therapies against epidermal growth factor receptor (EGFR) or anaplastic lymphoma kinase (ALK) have now been approved by the US Food and Drug Administration as part of the standard first-line treatment of NSCLC. Despite good initial responses, most patients develop resistance within 8-12 months and have disease progression.

HSP90 is a chaperone protein assisting other cellular proteins to fold properly, and stabilizes them against oxidative and heat stress, as well as helping with protein degradation. ${ }^{3}$ HSP90 is a weak ATPase with a very rapid turnover rate $(0.1 /$ minute in humans). Unlike ALK and EGFR NSCLC that is driven by oncogenic mutations, the activity of HSP90 is regulated by the binding of cochaperone molecules that induce conformational change in the HSP90. To date, over 20 cochaperone molecules have been identified. ${ }^{4}$

Recent insights from the Cancer Genome Atlas revealed that multiple malignancies overexpress or possess mutant kinases that depend on the HSP90-chaperone complex. ${ }^{5}$ HSP90 has been shown to stabilize various signaling molecules, such as PI3K and AKT proteins, thus inhibiting cellular apoptosis in cancerous cells (Figure 1). ${ }^{6}$ It also appears that HSP90 can act as a "protector" of unstable protein by-products of DNA mutations, such as v-SRC and mutant forms of $\mathrm{p} 53 .^{7}$ Relevant to NSCLC, mutant $E G F R,{ }^{8}$ ERBB $2,{ }^{9} M E T,{ }^{10}$ mutant $B R A F,{ }^{11}$ and the EML4-ALK translocation product ${ }^{12}$ are all HSP90-dependent proteins, the degradation of which leads to loss of tumor-cell viability in the corresponding adenocarcinoma subset. Expression of HSP90 has thus 


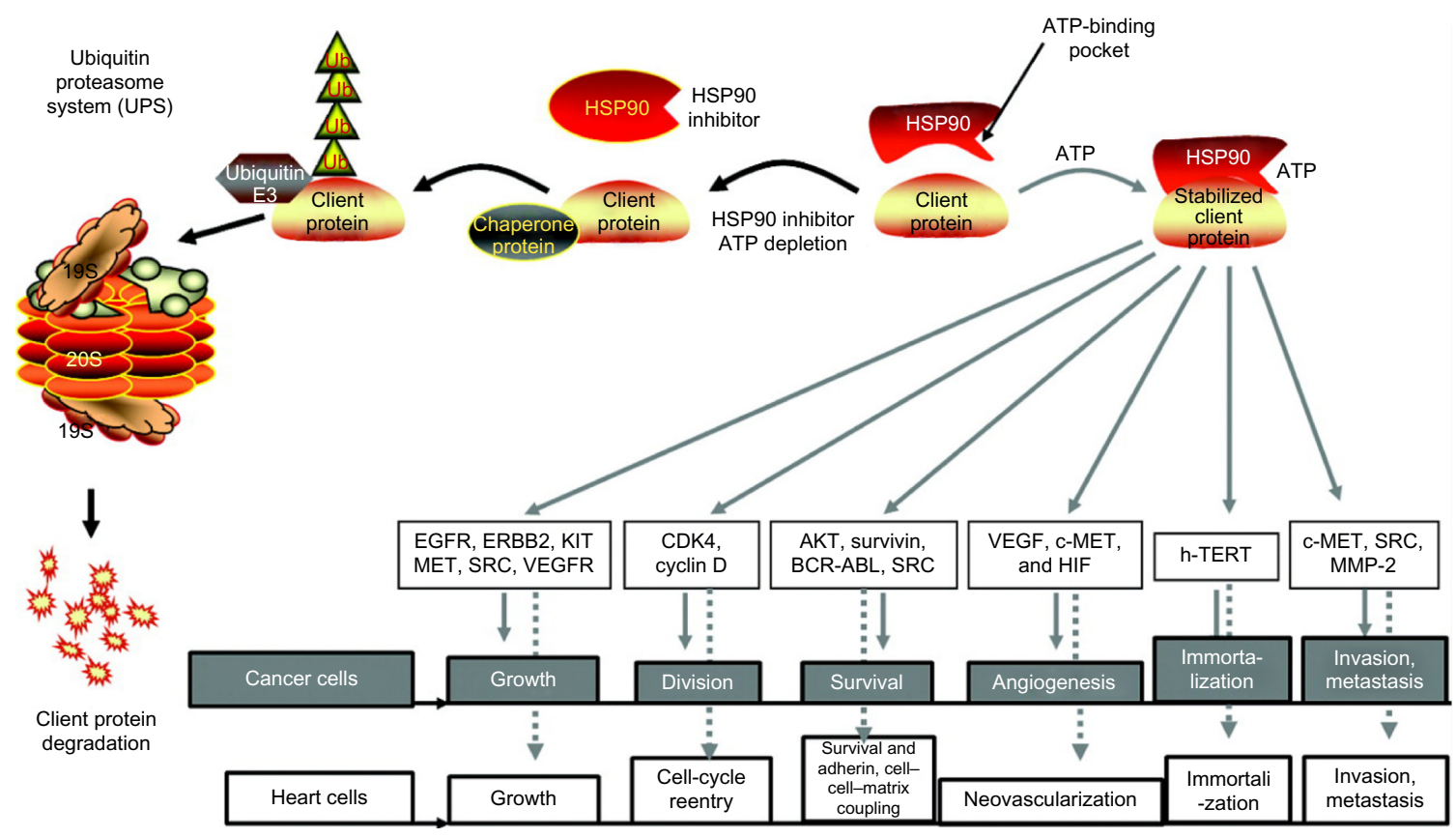

Figure I HSP90 and its chaperone proteins are involved in multiple cellular signaling pathways that regulate apoptosis and cell survival. Note: HSP90 inhibition results in ubiquitin-mediated degradation of its client protein. Abbreviation: ATP, adenosine triphosphate.

been shown to correlate with a worse clinical prognosis and to be correlated with resistance to chemo- and radiotherapy. ${ }^{13}$

The first class of HSP90 inhibitors to be characterized were the benzoquinone ansamycins, including geldanamycin and its derivatives 17-allylamino-17-demethoxygeldanamycin (17-AAG) and 17-dimethylaminoethylamino17-demethoxygeldanamycin (17-DMAG) ${ }^{14}$ They were derivatives of geldanamycin, which is an ansamycin antibiotic derived from a Streptomyces sp., and block ATP binding to the active site of HSP90. However, the clinical application of these drugs was limited by their poor pharmacokinetics and dynamics, including poor solubility, formulation problems, and potential multidrug efflux. ${ }^{15}$ As single agents, these molecules have shown only limited efficacy, thus pointing toward better results in combination therapy. ${ }^{16}$ In an effort to overcome these limitations, several second-generation synthetic HSP90 inhibitors representing multiple drug classes are currently under development.

The goal of this review is to present the data supporting the use of HSP90 inhibitors in NSCLC and to give an overview of the ongoing clinical trials involving new-generation HSP90 inhibitors. The summary of selected trials involving HSP90 inhibitors is presented in Table 1.

\section{Ganetespib (STA-9090)}

Ganetespib, also known as STA-9090, is a resorcinol derivative that binds the ATP-binding pocket of HSP90 and leads to its inhibition. Ganetespib acts by inducing $\mathrm{G}_{2} / \mathrm{M}$ cell-cycle phase arrest, resulting in apoptosis within 48 hours of treatment. It has also been shown to induce cell arrest in both erlotinibsensitive and erlotinib-resistant cell lines, including H1975 with the T790M gatekeeper mutation. ${ }^{17}$ Compared to the older generation 17-AAG, ganetespib has greater potency and potential efficacy against several NSCLC subsets, including those harboring EGFR or ERBB2 mutations. ${ }^{18}$ Treatment with ganetespib resulted in decreased downstream signaling through the PI3K-AKT-mTOR and RAF-MEK-ERK pathways.

In ALK-rearranged NSCLC cell lines, ganetespib induced loss of EML4-ALK expression and depletion of multiple oncogenic signaling proteins in ALK-driven NSCLC cells, leading to greater in vitro potency, superior antitumor efficacy, and prolonged animal survival compared with crizotinib monotherapy. ${ }^{19,20}$ Ganetespib also overcame multiple forms of crizotinib resistance, including secondary $A L K$ mutations. Cancer cells driven by ALK amplification and oncogenic rearrangements of the ROS1 and RET kinase genes were also sensitive to ganetespib exposure.

HSP90 inhibition with ganetespib has also resulted in decreased viability of $K R A S$-mutated cell lines by impacting downstream signaling through the mTOR and MEK pathways. ${ }^{21}$ It was superior to both AZD6244, an MEK inhibitor, and BEZ235, a PI3K/mTOR inhibitor when used as monotherapy, and also helped sensitize $K R A S$-mutated cell lines to cisplatin, pemetrexed, gemcitabine, and docetaxel. 
Table I Key completed trials involving HSP90 inhibitors

\begin{tabular}{|c|c|c|c|c|c|}
\hline Drug name & Trial information & Stratification & End point & ORR & Comments \\
\hline \multirow[t]{10}{*}{ Ganetespib } & NCT0I03I 225 & Cohort A: mutant RAS & PFS at 16 weeks & $4 \%$ (only seen in patients & Two deaths (one \\
\hline & $\mathrm{n}=99$ & Cohort B: mutant EGFR & Cohort A: $13.3 \%$ & with ALK translocation) & cardiac arrest and \\
\hline & Phase II & Cohort C: wild type & Cohort B: $5.9 \%$ & & one renal failure) \\
\hline & & & Cohort C: $13.9 \%$ & & \\
\hline & GALAXY-I & $\mathrm{LDH}$ & OS: HR 0.69 & $15 \%$ in the combination arm & Best response achieved \\
\hline & NCT0I348I26 & RAS mutation & PFS: HR 0.70 & $11 \%$ in stand-alone arm & in patients with $>6$ \\
\hline & $\mathrm{n}=255$ & Time since advanced disease & & & months since advanced \\
\hline & Phase II & Performance status & & & diagnosis (HR 0.4I) \\
\hline & Combination drug: & Smoking & & & \\
\hline & docetaxel & & & & \\
\hline \multirow[t]{4}{*}{ AUY922 } & NCT01922583 & EGFR-mutated & ORR and SD at & EGFR: $18 \%$ & SD achieved in some \\
\hline & $n=112$ & KRAS-mutated & 18 weeks & KRAS: $0 \%$ & crizotinib-resistant \\
\hline & Phase II & ALK-rearranged & Overall: $13 \%$ & ALK: $25 \%$ & patients \\
\hline & & Wild type & & Wild type: $13 \%$ & \\
\hline Retaspimycin & NCT0043I0I5 & EGFR wild-type & ORR of $7 \%$ & EGFR wild-type: $10 \%$ & Grade 3 liver \\
\hline \multirow[t]{2}{*}{ (IPI-504) } & $\mathrm{n}=76$ & EGFR-mutated & & EGFR-mutated: $4 \%$ & dysfunction in $11 \%$ \\
\hline & Phase II & & & & of patients \\
\hline
\end{tabular}

Abbreviations: ORR, overall response rate; PFS, progression-free survival; OS, overall survival; HR, hazard ratio; SD, stable disease; EGFR, epidermal growth factor receptor; ALK, anaplastic lymphoma kinase; LDH, lactate dehydrogenase.

HSP90 inhibitors have been shown to exert synergistic activity when combined with taxanes (paclitaxel and docetaxel), another $\mathrm{G}_{2} / \mathrm{M}$ cell-cycle arrest inhibitor. This combination has resulted in synergistic toxicity in both cell lines and animal xenograft models, resulting in more tumor regression than with taxanes alone. ${ }^{22}$

In the first published multicenter Phase II trial, ganetespib as monotherapy was assessed in previously treated patients with NSCLC with specific genotypic subtypes. ${ }^{23}$ A total of 99 patients were enrolled in three cohorts: cohort A $(\mathrm{n}=15$, mutant $E G F R), \mathrm{B}(\mathrm{n}=17$, mutant $K R A S)$, and $\mathrm{C}(\mathrm{n}=66$, no $E G F R$ or $K R A S$ mutations). Patients were treated with $200 \mathrm{mg} / \mathrm{m}^{2}$ ganetespib by intravenous infusion once-weekly for 3 weeks followed by 1 week of rest until disease progression. The primary end point of progressionfree survival (PFS) at 16 weeks was achieved in $13.3 \%$ of $E G F R$-mutated patients, $5.9 \%$ of $K R A S$-mutated patients, and $19.7 \%$ of wild-type patients; $4 \%$ of patients achieved partial response, all harboring the ALK translocation. Eight (8.1\%) patients experienced treatment-related serious adverse events, two of which (cardiac arrest and renal failure) resulted in death. The most common other adverse effects were diarrhea, fatigue, nausea, and anorexia.

Given early success in preclinical studies as combination therapy with taxanes, ganetespib was assessed in a Phase I trial in combination with docetaxel. The study, with 27 patients, defined the maximum tolerated dose (MTD) of ganetespib at $150 \mathrm{mg} / \mathrm{m}^{2}$ on days 1 and 15 of each 21-day cycle. ${ }^{24}$ The dose-limiting toxicities were febrile neutropenia and grade 4 neutropenia. Other adverse effects included anemia, fatigue, nausea, and diarrhea. This combination was further assessed in the Phase II GALAXY-1 trial comparing docetaxel to docetaxel plus ganetespib: ${ }^{25,26} 225$ patients with advanced NSCLC, one prior systemic therapy, and Eastern Cooperative Oncology Group performance status of $0 / 1$ were included. Docetaxel was given at $75 \mathrm{mg} / \mathrm{m}^{2}$ on day 1 of a 3-week cycle. In the experimental arm, docetaxel was given on day 1 and ganetespib at $150 \mathrm{mg} / \mathrm{m}^{2}$ on days 1 and 15. Patients were stratified by performance status, time since advanced disease diagnosis ( $\leq 6$ months vs $>6$ months), baseline lactate dehydrogenase (LDH) (elevated or normal), or smoking status. The rationale for including patients with elevated LDH was the correlation with tumor hypoxia in these patients attributable to HIF-1 $\alpha$, a client protein of HSP90. After 72 patients were enrolled, nonadenocarcinoma patients were excluded, given the lack of efficacy and excess bleeding risk. For the combination vs the monotherapy arm, the median number of cycles delivered was five vs four, and grade $3 / 4$ adverse events were neutropenia $38 \%$ vs $37 \%$, fatigue $4 \%$ vs $3 \%$, anemia $7 \%$ vs $6 \%$, diarrhea $3 \%$ vs $0 \%$, and fever with neutropenia $8 \%$ vs $2 \%$. In this study, the overall survival hazard ratio (HR) was 0.69 (95\% confidence interval $[\mathrm{CI}] 0.48-0.99, P=0.093)$, the PFS HR was 0.70 (95\% CI 0.53-0.94, $P=0.012$ ), and the overall response rate (ORR) was $15 \%$ vs $11 \%$, all favoring the combination arm. For patients that were enrolled $>6$ months after diagnosis of advanced NSCLC $(n=175,69 \%)$, a prespecified stratification factor, the overall survival HR was $0.41(95 \%$ CI $0.25-0.67, P=0.0009)$, the PFS HR was $0.47(95 \% \mathrm{CI}$ $0.32-0.69, P=0.0005)$, and the ORR was $16 \%$ vs $12 \%$. 
Given positive results from this study, multiple Phase III trials are under way to assess the efficacy of ganetespib in other adenocarcinomas, including breast cancer.

\section{AUY922}

AUY922 is a second-generation nongeldanamycin HSP90 inhibitor that is an isoxazole derivative. In preclinical studies, it has been shown to have clinical activity in lung cancer cells with MET- and AKL-mediated resistance. ${ }^{27}$ AUY 922 treatment effectively suppressed proliferation and induced cell death in both resistant cell lines by downregulating EGFR, MET, and AXL expression, which led to decreased AKT-pathway activation. AUY922 has also been shown to act as a radiosensitizer to cell lines with acquired resistance to EGFR inhibitors. ${ }^{28-30}$ These cell-line studies have been replicated in animals with NSCLC xenograft tumors with MET- and AXL-mediated resistance. ${ }^{27}$

A total of 101 patients were enrolled in the first-in-human dose-escalation study of AUY922 in patients with solid tumors. ${ }^{31}$ The MTD was not reached; however, because of concerns regarding visual toxicity, dose escalation beyond $70 \mathrm{mg} / \mathrm{m}^{2}$ was not attempted. Dose-limiting toxicities occurred in eight patients, and included diarrhea, asthenia/ fatigue, anorexia, atrial flutter, and visual symptoms. At $70 \mathrm{mg} / \mathrm{m}^{2}$, the AUY922 concentration achieved was consistent with active concentrations in a range of xenograft models, and that dose was recommended to be taken forward in Phase IB and Phase II studies. All 101 patients were evaluable for a clinical response, and of these, none had a complete or partial response by adapted Response Evaluation Criteria in Solid Tumors criteria.

The first Phase II trial evaluated AUY922 in 112 patients with NSCLC, $61 \%$ of which had received at least three lines of therapy. ${ }^{32}$ Patients were stratified in to four groups: $E G F R$-mutated, KRAS-mutated, ALK-rearranged, and wildtype NSCLC. Mean duration of exposure was 9 weeks. In a preliminary report presented at the American Society of Clinical Oncology 2012 conference, partial responses were seen in 13 out of 101 (13\%) patients, two out of eight (25\%) ALK-rearranged patients, six out of 33 (18\%) EGFR-mutated patients, four out of $30(13 \%)$ wild-type patients, zero out of $26(0 \%) K R A S$-mutated patients, and one out of four $(25 \%)$ patients of unknown status. In ALK-rearranged patients, responses were seen in crizotinib-naïve patients, and stable disease was seen with tumor shrinkage in crizotinib-resistant patients. The most frequent adverse events were diarrhea (73\%), visual disturbances ( $71 \%$ ), and nausea (43\%).
These data were updated at the 2013 American Society of Clinical Oncology Annual Meeting, with a focus on EGFRmutated patients. Data for 16 patients with acquired EGFR tyrosine-kinase inhibitor (TKI) resistance were presented, seven having tested positive for EGFR T790M at rebiopsy. The ORR was two out of 16 (13\%), and both patients with partial responses had the gatekeeper mutation.

Another Phase II trial looked at using AUY922 in patients with acquired resistance to erlotinib; ${ }^{33} 25$ patients were evaluated, and the median time to TKI resistance was 11 months. Ten (40\%) patients had EGFR T790M identified by tumor rebiopsy. The ORR was four out of 25 (16\%). Three out of four patients with partial response had EGFR T790M mutation. An additional four patients had stable disease for at least 8 weeks. Adverse events reported in $\geq 20 \%$ of patients were diarrhea, fatigue, myalgia, nausea, mucositis, and night blindness; $68 \%$ (17 out of 25) experienced night blindness (grade 1-2 only), and three patients came off the study due to eye-related toxicity.

AUY922 has also been combined with trastuzumab in patients with HER2-amplified or HER2-mutated NSCLC; 34 55 patients with HER2 amplification, 34 patients with HER2 overexpression, and seven patients with HER 2 mutation were treated. Final results from this study are pending publication.

\section{Retaspimycin (IPI-504)}

Retaspimycin or IPI-504 is a water-soluble derivate of 17-AAG that causes less liver toxicity and has shown activity in NSCLC. In preclinical studies, great responses have been obtained with retaspimycin, especially in ALK-rearranged cell lines. Treatment of H3122 ALK-rearranged cell lines with retaspimycin resulted in degradation of the fusion protein in $<3$ hours, making it a much more sensitive target than EGFR or HER2. ${ }^{12}$

The first multicenter Phase II study of retaspimycin enrolled 76 patients with EGFR-mutated lung cancer that had progressed on TKI therapy. ${ }^{35}$ The ORR was a disappointing five out of $76(7 \%)$ in the overall study population, $10 \%$ in patients with EGFR wild-type, and $4 \%$ in patient with EGFR mutations, with a median PFS of 2.86 months. However, among three patients with ALK rearrangements, two had partial responses and had prolonged stable disease of over 7 months' duration. Grade 3 hepatitis was observed in $12 \%$ of patients. The most common adverse effects were fatigue, nausea, and diarrhea.

Retaspimycin has also been evaluated in combination with taxanes in patients with metastatic NSCLC in an expansion of 
Table 2 Ongoing clinical trials with HSP90 inhibitors

\begin{tabular}{|c|c|c|c|c|}
\hline Drug & Clinical trial number & Phase & Adjunct drug & Mutation selection \\
\hline \multirow[t]{7}{*}{ Ganetespib } & GALAXY-2 (NCT0I798485) & III & Docetaxel & - \\
\hline & NCT0I348I 26 & $\|\mathrm{IB} /\| \mathrm{I}$ & Docetaxel & - \\
\hline & NCT0I03I 225 & II & - & - \\
\hline & NCT0I5620I5 & II & - & - \\
\hline & NCT0I579994 & I & Crizotinib & ALK \\
\hline & NCT0226|805 (small-cell lung cancer) & 1 & Doxorubicin & - \\
\hline & NCT0I590I60 (mesothelioma) & $\mathrm{I} / \mathrm{II}$ & - & \\
\hline \multirow[t]{9}{*}{ AUY922 } & NCT01922583 & II & - & \\
\hline & NCT0I854034 & II & - & EGFR exon 20 \\
\hline & NCT0I752400 & II & - & ALK \\
\hline & NCT0I646I25 & II & Vs pemetrexed/docetaxel & EGFFR \\
\hline & NCTOII 24864 & ॥ & - & EGFR/ALK/W ${ }^{+}$ \\
\hline & NCT02276027 & ॥ & - & \\
\hline & NCT0I 259089 & $1 / I I$ & Erlotinib & EGFR \\
\hline & NCT0I772797 & IB & LDK378 & ALK \\
\hline & NCT0I 784640 & 1 & Pemetrexed & - \\
\hline \multirow[t]{4}{*}{ IPI-504 } & NCT0I 362400 & II & Docetaxel & - \\
\hline & NCT0I 228435 & II & - & ALK \\
\hline & NCT0I427946 & $\mathrm{IB} / \mathrm{II}$ & Everolimus & - \\
\hline & NCT0043I0I5 & $1 / I I$ & - & - \\
\hline ATI 3387 & NCT0I7I22I7 & $1 / I 1$ & Crizotinib & ALK \\
\hline
\end{tabular}

Abbreviations: ALK, anaplastic lymphoma kinase; EGFR, epidermal growth factor receptor.

a Phase IB trial; ${ }^{36} 23$ patients with pathologically confirmed metastatic NSCLC, all having received one to two prior chemotherapy regimens without prior docetaxel, were enrolled. Docetaxel $75 \mathrm{mg} / \mathrm{m}^{2} \mathrm{IV}$ was given once every 3 weeks, while retaspimycin $300 \mathrm{mg} / \mathrm{m}^{2}$ was administered intravenously once per week. Six out of the 23 (26\%) patients had a partial response, including three of seven patients with squamous cell carcinoma. The most common reported adverse effects were fatigue, diarrhea, neutropenia, and anemia.

\section{AT 13387}

AT13387 is a potent second-generation nonansamycin HSP90 inhibitor. It has been shown to have effect in NSCLC cell lines, as well as mouse xenograft models. ${ }^{37}$ Its long duration of action has enabled once-weekly dosing. ${ }^{38}$ AT 13387 has also shown clinical efficacy in ALK-rearranged cell lines, as well as mouse xenograft models injected with those cells. ${ }^{39}$

In the first-in-human Phase I dose-escalation study, AT13387 was evaluated in 62 patients with advanced solid tumors. The drug was administered in both a twice-weekly and once-weekly regimen. One dose-limiting visual disturbance occurred at $120 \mathrm{mg} / \mathrm{m}^{2}$ twice weekly, thus establishing the MTD for this regimen. For the once-weekly regimen, no formal dose-limiting toxicity occurred; however, multiple moderately severe toxicities, including diarrhea, nausea, vomiting, fatigue, and systemic infusion reactions, led to selection of $260 \mathrm{mg} / \mathrm{m}^{2}$ as the recommended Phase II dose. The drug was administered for 3 weeks on a 4-week cycle.

\section{Conclusion}

The HSP90 inhibitors are a diverse group of molecules with a wide range of activity in solid tumors, with ongoing trials presented in Table 2. These compounds have demonstrated significant potential in NSCLC in both preclinical and human trials. They can also act as chemo- and radiosensitizers, as well as help circumvent acquired resistance to standard targeted therapies. The advantage of using HSP90 inhibitors is their ability to target multiple oncogenes at the same time. The GALAXY-2 study (NCT01798485), the first Phase III trial of HSP90 inhibitors in NSCLC, is ongoing. A previous study reported single amino acid mutations in the HSP90 molecule associated with in vivo resistance to HSP90 inhibitors. ${ }^{40}$ Further studies are required to identify additional predictive biomarkers of sensitivity and resistance to HSP90 inhibitors in humans.

\section{Disclosure}

The authors report no conflicts of interest in this work.

\section{References}

1. Siegel RL, Miller KD, Jemal A. Cancer statistics, 2015. CA Cancer J Clin. 2015;65(1):5-29. 
2. Kumarakulasinghe NB, van Zanwijk N, Soo RA. Molecular targeted therapy in the treatment of advanced stage non-small cell lung cancer (NSCLC). Respirology. 2015;20(3):370-378.

3. Mahalingam D, Swords R, Carew JS, Nawrocki ST, Bhalla K, Giles FJ. Targeting HSP90 for cancer therapy. Br J Cancer. 2009;100(10): 1523-1529.

4. Li J, Buchner J. Structure, function and regulation of the Hsp90 machinery. Biomed J. 2013;36(3):106-117.

5. Schwartz H, Scroggins B, Zuehlke A, et al. Combined HSP90 and kinase inhibitor therapy: insights from the Cancer Genome Atlas. Cell Stress Chaperones. 2015;20(5):729-741.

6. Sawai A, Chandarlapaty S, Greulich H, et al. Inhibition of Hsp90 down-regulates mutant epidermal growth factor receptor (EGFR) expression and sensitizes EGFR mutant tumors to paclitaxel. Cancer Res. 2008;68(2):589-596.

7. Calderwood SK, Khaleque MA, Sawyer DB, Ciocca DR. Heat shock proteins in cancer: chaperones of tumorigenesis. Trends Biochem Sci. 2006;31(3):164-172.

8. Shimamura T, Lowell AM, Engelman JA, Shapiro GI. Epidermal growth factor receptors harboring kinase domain mutations associate with the heat shock protein 90 chaperone and are destabilized following exposure to geldanamycins. Cancer Res. 2005;65(14):6401-6408.

9. Xu W, Soga S, Beebe K, et al. Sensitivity of epidermal growth factor receptor and ErbB2 exon 20 insertion mutants to Hsp90 inhibition. Br J Cancer. 2007;97(6):741-744.

10. Wang S, Pashtan I, Tsutsumi S, Xu W, Neckers L. Cancer cells harboring MET gene amplification activate alternative signaling pathways to escape MET inhibition but remain sensitive to Hsp90 inhibitors. Cell Cycle. 2009;8(13):2050-2056.

11. da Rocha Dias S, Friedlos F, Light Y, Springer C, Workman P, Marais R. Activated B-RAF is an Hsp90 client protein that is targeted by the anticancer drug 17-allylamino-17-demethoxygeldanamycin. Cancer Res. 2005;65(23):10686-10691.

12. Normant E, Paez G, West KA, et al. The Hsp90 inhibitor IPI-504 rapidly lowers EML4-ALK levels and induces tumor regression in ALK-driven NSCLC models. Oncogene. 2011;30(22):2581-2586.

13. Garcia-Carbonero R, Carnero A, Paz-Ares L. Inhibition of HSP90 molecular chaperones: moving into the clinic. Lancet Oncol. 2013;14(9): e358-e369.

14. Neckers L, Ivy SP. Heat shock protein 90. Curr Opin Oncol. 2003;15(6): 419-424.

15. Powers MV, Workman P. Targeting of multiple signalling pathways by heat shock protein 90 molecular chaperone inhibitors. Endocr Relat Cancer. 2006;13 Suppl 1:S125-S135.

16. Biamonte MA, Shi J, Hong K, et al. Orally active purine-based inhibitors of the heat shock protein 90. J Med Chem. 2006;49(2):817-828.

17. Ying W, Du Z, Sun L, et al. Ganetespib, a unique triazolone-containing Hsp90 inhibitor, exhibits potent antitumor activity and a superior safety profile for cancer therapy. Mol Cancer Ther. 2012;11(2):475-484.

18. Shimamura T, Perera SA, Foley KP, et al. Ganetespib (STA-9090), a nongeldanamycin HSP90 inhibitor, has potent antitumor activity in in vitro and in vivo models of non-small cell lung cancer. Clin Cancer Res. 2012;18(18):4973-4985.

19. Sang J, Acquaviva J, Friedland JC, et al. Targeted inhibition of the molecular chaperone Hsp90 overcomes ALK inhibitor resistance in non-small cell lung cancer. Cancer Discov. 2013;3(4):430-443.

20. Richards MW, Law EW, Rennalls LP, et al. Crystal structure of EML1 reveals the basis for Hsp90 dependence of oncogenic EML4-ALK by disruption of an atypical $\beta$-propeller domain. Proc Natl Acad Sci USA. 2014;111(14):5195-5200.

21. Acquaviva J, Smith DL, Sang J, et al. Targeting KRAS-mutant non-small cell lung cancer with the Hsp90 inhibitor ganetespib. Mol Cancer Ther. 2012;11(12):2633-2643.

22. Proia DA, Sang J, He S, et al. Synergistic activity of the Hsp90 inhibitor ganetespib with taxanes in non-small cell lung cancer models. Invest New Drugs. 2012;30(6):2201-2209.
23. Socinski MA, Goldman J, El-Hariry I, et al. A multicenter phase II study of ganetespib monotherapy in patients with genotypically defined advanced non-small cell lung cancer. Clin Cancer Res. 2013;19(11): 3068-3077.

24. Kauh JS, Harvey RD, Owonikoko TK, et al. A phase I and pharmacokinetic study of multiple schedules of ganetespib (STA-9090), a heat shock protein 90 inhibitor, in combination with docetaxel for subjects with advanced solid tumor malignancies. J Clin Oncol. 2012; 30 Suppl:3094.

25. Ramalingam SS, Goss GD, Andric ZG, et al. A randomized study of ganetespib, a heat shock protein 90 inhibitor, in combination with docetaxel versus docetaxel alone for second-line therapy of lung adenocarcinoma (GALAXY-1). J Clin Oncol. 2013;31 Suppl: CRA8007.

26. Ramalingam SS, Shapiro G, Hirsh V, etal.GALAXY-1: randomized phase II study of docetaxel with or without ganetespib in advanced lung adenocarcinoma results in biomarker sub-groups and all adenocarcinoma patients. J Thorac Oncol. 2013;8 Suppl 2:S139.

27. Choi YJ, Kim SY, So KS, et al. AUY922 effectively overcomes METand AXL-mediated resistance to EGFR-TKI in lung cancer cells. PLoS One. 2015;10(3): $\mathrm{e} 0119832$.

28. Hashida S, Yamamoto H, Shien K, et al. Hsp90 inhibitor NVP-AUY922 enhances the radiation sensitivity of lung cancer cell lines with acquired resistance to EGFR-tyrosine kinase inhibitors. Oncol Rep. 2015;33(3): 1499-1504.

29. Djuzenova CS, Blassl C, Roloff K, et al. Hsp90 inhibitor NVP-AUY 922 enhances radiation sensitivity of tumor cell lines under hypoxia. Cancer Biol Ther. 2012;13(6):425-434.

30. Schilling D, Bayer C, Li W, Molls M, Vaupel P, Multhoff G. Radiosensitization of normoxic and hypoxic h1339 lung tumor cells by heat shock protein 90 inhibition is independent of hypoxia inducible factor-1 $\alpha$. PLoS One. 2012;7(2):e31110.

31. Sessa C, Shapiro GI, Bhalla KN, et al. First-in-human phase I doseescalation study of the HSP90 inhibitor AUY922 in patients with advanced solid tumors. Clin Cancer Res. 2013;19(13):3671-3680.

32. Garon EB, Moran T, Barlesi F, et al. Phase II study of the HSP90 inhibitor AUY922 in patients with previously treated, advanced non-small cell lung cancer (NSCLC). J Clin Oncol. 2012;30 Suppl:7543.

33. Yu H, Johnson M, Urman A, et al. A phase II study of HSP90 inhibitor AUY922 and erlotinib (E) in patients (pts) with EGFR-mutant lung cancer and acquired resistance (AR) to EGFR tyrosine kinase inhibitors (EGFR TKIs). J Thorac Oncol. 2013;8 Suppl 2:S597.

34. Nogova L, Gardizi M, Bos MC, et al. TRY: A phase II study to evaluate safety and efficacy of combined trastuzumab and AUY 922 in advanced non-small-cell lung cancer (NSCLC) with HER2 overexpression or amplification or mutation. J Clin Oncol. 2014;32 Suppl 5:8109.

35. Sequist LV, Gettinger S, Senzer NN, et al. Activity of IPI-504, a novel heat-shock protein 90 inhibitor, in patients with molecularly defined non-small-cell lung cancer. J Clin Oncol. 2010;28(33): 4953-4960.

36. Riely GJ, Gettinger SN, Stoller RG, et al. Safety and activity of IPI-504 (retaspimycin hydrochloride) and docetaxel in pretreated patients (pts) with metastatic non-small-cell lung cancer (NSCLC). J Clin Oncol. 2011;29 Suppl:7516.

37. Hearn K, Smith T, Lewis J, et al. The Hsp90 inhibitor, AT13387, displays single agent activity in erlotinib-sensitive and -resistant models of EGFR activated NSCLC. J Thorac Oncol. 2013;8 Suppl 2:S464.

38. Graham B, Curry J, Smyth T, et al. The heat shock protein 90 inhibitor, AT13387, displays a long duration of action in vitro and in vivo in non-small cell lung cancer. Cancer Sci. 2012;103(3):522-527.

39. Smyth T, Munck J, Rodriguez-Lopez A, et al. Combining the Hsp90 inhibitor, AT13387, with crizotinib improves response in an ALK positive model of NSCLC. J Thorac Oncol. 2013;8 Suppl 2:S163.

40. Zurawska A, Urbanski J, Matuliene J, et al. Mutations that increase both Hsp90 ATPase activity in vitro and Hsp90 drug resistance in vivo. Biochim Biophys Acta. 2010;1803(5):575-583. 
Lung Cancer: Targets and Therapy

Dovepress

\section{Publish your work in this journal}

Lung Cancer: Targets and Therapy is an international, peer-reviewed, open access journal focusing on lung cancer research, identification of therapeutic targets and the optimal use of preventative and integrated treatment interventions to achieve improved outcomes, enhanced survival and quality of life for the cancer patient Specific topics covered in the journal include: Epidemiology, detection and screening Cellular research and biomarkers; Identification of biotargets and agents with novel

Submit your manuscript here: http://www.dovepress.com/lung-cancer-targets--therapy-journal

mechanisms of action; Optimal clinical use of existing anticancer agents, including combination therapies; Radiation and surgery; Palliative care; Patient adherence, quality of life, satisfaction; Health economic evaluations. The manuscript management system is completely online and includes a very quick and fair peer-review system. Visit http://www.dovepress.com/testimonials.php to read real quotes from published authors.
system. 